\title{
Oil Spill Visualization Based on the Numeric Simulation of Tidal Current
}

\author{
Feng Yu, Yong Yin
}

\section{Key Laboratory of Marine Simulation and Control, Dalian Maritime University}

\begin{abstract}
This paper proposes an approach to implement the 3D visualization of oil spill based on tidal hydrodynamic model. It simulates tidal current of $\mathrm{M}_{2}$ component tide in Jiaozhou Bay. The simulation results conform to the tidal theory and probably conform to the flow measurement report of crude oil pier Phase III at Qingdao Harbor. Based on tidal current and eye-point related adaptive ocean surface mesh model, by analyzing the drift and diffusion mathematical models of oil spill on the sea, the dynamic visualization of drift and diffusion course of oil on the sea were implemented, the visualization result is satisfactory.
\end{abstract}

Index Terms - Tidal Current, Numeric Simulation, Texture Blending, Oil Spill Visualization.

\section{INTRODUCTION}

Marine Search and Rescue Simulator (shorted for MSRS) is a kind of Man-In-the-Loop-Simulations system that can be used for integrative maritime search and rescue training. The oil spill emergency response information system is the important component of MSRS. As part of MSRS research in the institute we belong to, we realize 3D visualization of oil spill based on physical model and texture mapping. In order to visualize oil spill, the hydrodynamic condition is the first to be considered. Furthermore, the numeric simulation is an effective and economical method to simulate tidal current.

\section{NUMERIC CALCULATION OF TIDAL WAVES}

Blumberg and his research team build up the Princeton Ocean Model [1], it is a three-dimensional baroclinic ocean model based on the original equation. Continental shelf topography is conveniently used in the model because of $\sigma$ coordinate. So POM model is widely used in the estuary and coastal region's tidal current simulation. During 2005-2006 the third generation prediction product is developed by G. Fang et al [2]. This product covered the entire China adjacent seas, with a resolution of $5^{\prime} \times 5^{\prime}$ and 10 levels in the vertical. In the field of numeric simulation of tidal current, the monthly and annual mean freshwater, heat and salt transport through the open boundaries of the South and East China Seas derived from a variable-grid global ocean circulation model is proposed by G. Fang [3]. A self adaptive mesh is used in the East China Sea for the first time by X. Zhou [4]. The grid points at boundary are corresponding to the actual boundary,

Manuscript Received on 08122008

E-Mail: fenger_1012@163.com and the grid points are denser at the continental slope of East Sea than elsewhere. From earlier studies, we know that, the offshore and coastal zones are mainly broad-shallow water, the horizontal measure is bigger than vertical measure, so the 2D tidal hydrodynamic models simplified from 3D models exactly show tidal characters. We adopt a simplified 2D model to simulate tidal current in Jiaozhou Bay.

\subsection{Two-dimensional Hydrodynamic Model}

The hydrodynamic model is presupposed as follows: fluid is incompressible viscous; pressure is distribution of hydrostatic pressure; the change of time and space in vertical direction $\left(\frac{\partial \omega}{\partial t}, \frac{\partial \omega}{\partial x}, \frac{\partial \omega}{\partial y}, \frac{\partial \omega}{\partial z}\right)$ can be neglected; horizontal mixed term is smaller than vertical mixed term, let it be a negligible quantity. It can be described as follows:

$\frac{\partial \zeta}{\partial t}+\frac{\partial[(\zeta+h) u]}{\partial x}+\frac{\partial[(\zeta+h) v]}{\partial y}=0$

$\frac{\partial u}{\partial t}+u \frac{\partial u}{\partial x}+v \frac{\partial u}{\partial y}+g \frac{\partial \zeta}{\partial x}=-g \frac{u \sqrt{u^{2}+v^{2}}}{(\zeta+h) c^{2}}+f v$

$\frac{\partial v}{\partial t}+u \frac{\partial v}{\partial x}+v \frac{\partial v}{\partial y}+g \frac{\partial \zeta}{\partial y}=-g \frac{v \sqrt{u^{2}+v^{2}}}{(\zeta+h) c^{2}}-f u$

Where $u, v$ are velocity of eastern component and northern component; $\zeta$ is sea level; $t$ is time; $h$ is undisturbed water depth; $c$ is chezy coefficient, $c=1 / n *(\zeta+h)^{1 / 6}, n$ is manning roughness, taken as 0.0036 in the present computation; $f$ is coriolis force coefficient, $f=2 \omega \sin \varphi, \omega$ is rotational angular velocity of the earth, $\varphi$ is latitude.

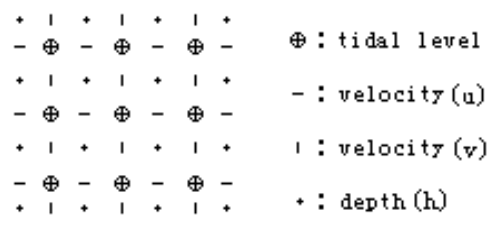

Fig. 1. Difference mesh

We adopts ADI method to discrete this 2D model. ADI method is a finite-difference method which is proposed by Peaceman [5]. The method halves a time step, in the first half, parameters along $x$ axis are represented by implicit equations, parameters along $y$ axis are represented by explicit equations; in the second half, parameters along $y$ axis are represented by implicit equations, parameters along $x$ axis are represented by explicit equations. The numeric calculation mesh used in ADI 
method can be described as follows:

\subsection{Boundary Condition of Tidal Waves Calculation}

The line between Tuandao and Houchawan in Jiaozhou Bay is set as open boundary. The water-land boundary except open boundary is set as closed boundary, the values of sea level and flow velocity are 0 . The enforced sea level formula [6] is as follows:

$$
\zeta_{(i, j)}(k)=H_{(i, j)} \cos \left(\sigma k-g_{(i, j)}\right) \quad k=0,1 \ldots N-1
$$

Where $H, g$ are tidal harmonic constants, $H$ is amplitude, $g$ is the phase-lag; $N$ is the number of time steps in one tidal period, providing a enforced sea level alternate half a time step; $\quad \sigma$ is the angular frequency of the component tide.

\subsection{Tidal Waves Numeric Calculation and Discussions}

We only analyze $\mathrm{M}_{2}$ component tide, it is semidiurnal tide, owing to the minimum impact of other component tides in Jiaozhou Bay. We use $\mathrm{VC}++6.0$ to compile program. Harmonic constants of $\mathbf{M}_{2}$ component tide are got by analyzing the flow measurement report of crude oil pier Phase III at Qingdao Harbor. The mesh grid is rectangular grid, there are 1960 points and 1906 units in the computation region, the space between rectangle grid - $\Delta x$ and $\Delta y$ are $400 \mathrm{~m}$, time step is $2 \mathrm{sec}$. The number of time steps of $\mathrm{M}_{2}$ component tide in one tidal period is 22350, water depths come from 12351 sea charts, angular speed of $\mathrm{M}_{2}$ component tide is $28.9841^{\circ} / \mathrm{h}$. Initial value $\zeta=u=v=0$, let the values of $\zeta, u, v$ which are calculated after the first tidal period be the initial values of the second tidal period calculation, and the rest may be deduced by analogy. It can be steady after 4 or 5 tidal periods. The sea level, flow velocity and flow direction can be calculated by these formulas:

$$
\begin{aligned}
& u_{(i, j)}(k)=\left[u_{(i+1 / 2, j)}(k)+u_{(i-1 / 2, j)}(k)\right] / 2 \\
& v_{(i, j)}(k)=\left[v_{(i, j+1 / 2)}(k)+v_{(i, j-1 / 2)}(k)\right] / 2 \\
& w_{(i, j)}(k)=\left[u_{(i, j)}^{2}(k)+v_{(i, j)}^{2}(k)\right]^{1 / 2} \\
& \theta_{(i, j)}(k)=\arctan \left[u_{(i, j)}(k) / v_{(i, j)}(k)\right]
\end{aligned}
$$

Where $u(k)$ is the eastern component flow velocity; $v(k)$ is the northern component flow velocity; $w(k)$ is scalar quantity of flow velocity; $\theta(k)$ is flow director.

Tidal current at any time can be obtained by this calculation. We use Matlab7.1 to create flow field figures, they separately describe the tidal current at 7:00, 10:00, 13:00 and 16:00 in one day.

In the flow field figure, there are not only the flow direction and velocity; there are co-amplitude lines of M2 component tide that are given in meter. We can clearly see the tidal current status. We analyze the water in the inlet region of Jiaozhou Bay. Fig. 2 and Fig. 3 show that the flows in the inlet region are fast-moving, the flow direction is west-north, it is at the flood, water swarm into Jiaozhou Bay. The co-amplitude lines show that the amplitude in the northern region of Jiaozhou Bay is bigger than other regions. The amplitude of every point in Jiaozhou Bay change through time.

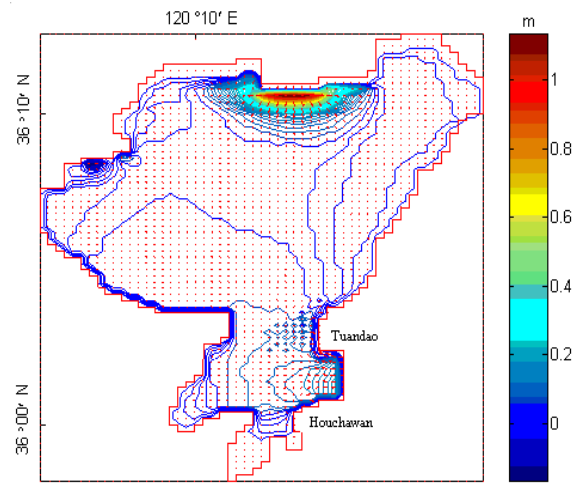

Fig. 2. Tidal current at 7:00.

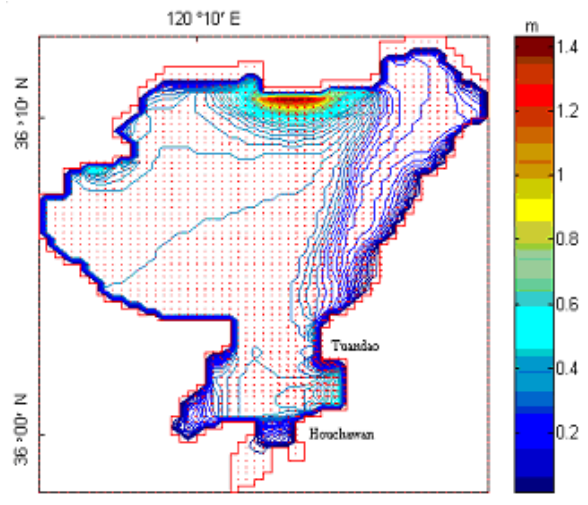

Fig. 3. Tidal current at 10:00.

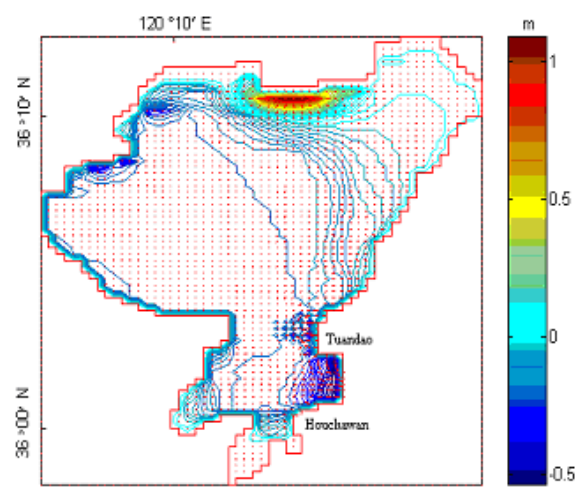

Fig. 4. Tidal current at 13:00.

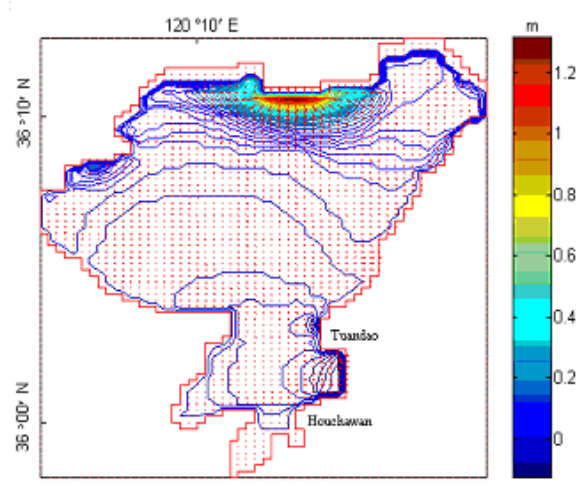

Fig. 5. Tidal current at 16:00 
Fig. 4 and Fig. 5 show that the flow direction is south-east, it is at the ebb, water swarm out of Jiaozhou Bay.

Given northern flow direction is $0^{\circ}$, eastern direction is $90^{\circ}$. According to the calculation results, selecting point $\mathrm{A}(\mathrm{N}$ $36^{\circ} 2.515^{\prime}$, E $120^{\circ} 14.556^{\prime}$ ), the sea level, flow velocity and flow direction figure is as follows, it shows points A appearing two times low tides and high tides in 24 hours. The flow direction belongs to anti-clockwise rotating flow, its characters accord with the characters of $\mathrm{M}_{2}$ component tide.

Sea level and flow velocity calculated relates to boundary condition, parameters and water depth adoption. Time step is an important parameter, program will diverge if it is too long, and water depth and space between mesh knots directly affect its adoption.
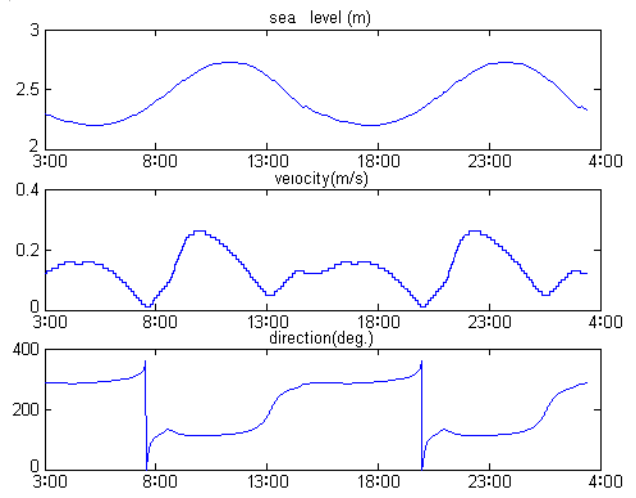

Fig. 6. Sea level, velocity and direction of flow figure (mean sea level is $2.39 \mathrm{~m})$.

Fig. 6 shows the maximum velocity at flood tide is $0.29 \mathrm{~m} / \mathrm{s}$ and ebb tide is $0.12 \mathrm{~m} / \mathrm{s}$, the maximum sea level is $2.7 \mathrm{~m}$, the minimum sea level is $2.1 \mathrm{~m}$. When flow is changing from flood tide time to ebb tide time or from ebb tide time to flood tide time, flow direction change a lot. To compare the sea level values calculated from this paper and the values from the flow measurement report of crude oil pier Phase III at Qingdao Harbor at point A, the contradistinctive figure is as follows:

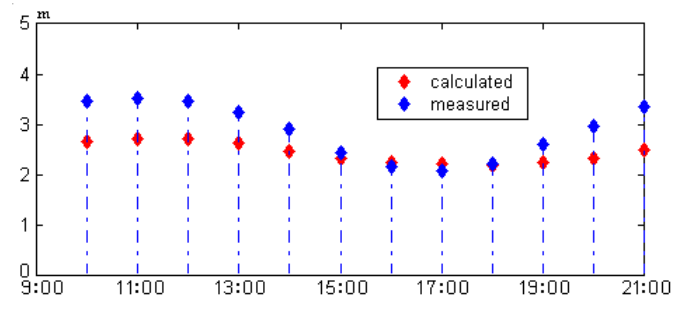

Fig. 7. Sea level contradistinctive figure.

By analyzing sea level values in Fig. 7, the values calculated in this paper conform to tidal theory and conform probably to the measured values from the flow measurement report of crude oil pier Phase III at Qingdao Harbor.

\section{3D VISUALIZATION OF OIL SPILL}

There are many research findings of oil spill on the sea, like geographical information system of marine oil spill emergency response which developed by Y. Liu [7]. These researches forecast the tracks of oil, but mostly only do the $2 \mathrm{D}$ display not 3D display, so the focus of our work is 3D display.

\subsection{Ocean Wave Model}

Virtual ocean environment is important expression of real feeling in navigation simulation system. In order to quickly realize virtual ocean environment in real-time, we use an eye-point related adaptive ocean surface mesh model [8]. When mesh resolution is $80 \times 60$, frame velocity is higher than $45 \mathrm{fps}$. It dynamically changes the dimensional position of the sea based on the position of eye-point. In navigation simulation system, simulation of ocean waves is an important technology. We think of the surface gravity wave which is caused by wind, it is called irregular long-crested wave in deep water. Irregular long-crested wave may be added up by a lot of regular long-crested waves with different amplitude and wavelength, so instantaneous wave height is as follows:

$\zeta=H_{\text {Tide }}+\sum_{i=1}^{n} \zeta_{a i} \cos \left[k_{i}(x \sin \theta+y \cos \theta)+\omega_{i} t+\varphi_{i}\right]$

Where $H_{\text {Tide }}$ is tidal height; $g$ is acceleration of gravity; $\theta$ is propagation direction of wave, $\theta \in[0,2 \pi] ; t$ is time; $\varphi_{i}$ is the phase angle; $k_{i}$ is wave number, gained from dispersion relation.

\subsection{Oil Drift and Diffusion Mathematical Models}

The theoretical-experiential formula [9] of oil diffusion falls into three parts: gravity diffusion, glutinosity diffusion and surface tension diffusion. It is described as follows:

$$
\begin{aligned}
& r(t)=K_{1}\left(\Delta g V t^{2}\right)^{1 / 4} \\
& r(t)=K_{2}\left(\Delta g V t^{2 / 3} / v^{1 / 2}\right)^{1 / 6} \\
& r(t)=K_{3}\left(\sigma^{2} t^{3} / \rho^{2} v\right)^{1 / 4}
\end{aligned}
$$

Where $\Delta g=\left(\rho-\rho_{0}\right) * g / \rho, \rho_{0}$ and $\rho_{w}$ are the density of oil and water; $r(t)$ is oil diffusion scale; $\sigma=\delta_{w a}-\delta_{a o}-\delta_{o w}$, $\delta_{w a}, \delta_{a o}, \delta_{o w}$ are surface tension between water and air, oil and air, oil and water; $v$ is the kinematic viscosity coefficient of water; $t$ is time; $V$ is oil' volume; $K_{1}, K_{2}$, $K_{3}$ are experiential coefficient, taken as $1.14,1.0$ and 1.5 in the present computation.

The drift velocity of any point on the edge of oil at different time and in different directions can be described as follows:

$-\vec{u}_{t}(t, \theta)=\vec{u}_{c}+K_{2} \vec{u}_{10}$

Where $\vec{u}_{c}$ is velocity vector of flow on the sea surface, which is obtained by calculation from (1) (3); $\vec{u}_{10}$ is wind speed vector at $10 \mathrm{~m}$ altitude from the sea surface; $K_{2}$ is wind drift coefficient, taken as 0.035 in the present computation. The process figure of oil drift and diffusion is as follows:

In Fig. 8, the small circle is oil in initial status; the big one is the status after $20 \mathrm{~min}$. It shows oil travels some distance in $20 \mathrm{~min}$ and its shape is changed, it is bigger. The ashen curve $\mathrm{L}$ between point 01 and 02 is the track of oil; o1 and 02 are the centre of circle; $\mathrm{r} 1$ and $\mathrm{r} 2$ are semi diameters; $\Delta x$ and $\Delta y$ are the horizontal and vertical spaces. The oil diffusion scale, drift velocity and direction are obtained by calculation from this subsection, so next, we can visualize oil. 


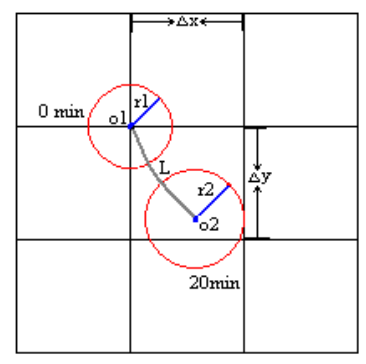

Fig. 8. Oil drift and diffusion process.

\subsection{D Visualization Result}

We proposes an approach based on physical model and texture mapping to implement the $3 \mathrm{D}$ visualization of oil spill, the computer we use is installed of NVIDIA Quadro 4400G GPU. The program is called OpenGL instructions and OpenGVS APIs to render 16 waves series. The dynamic simulation of drift and diffusion course of oil spill on the sea is implemented by means of texture mapping mode. Because the displacement of oil drift course is within $400 \mathrm{~m}$ within half an hour according to (13) in this experience, so we only see the oil move in visual form. First Importing oil spill time, oil variety, wind speed and direction et al, then using different oil textures to render. This simulation system can visualize oil drift and diffusion based on the different input data. Oil can move with wave model and melt into one another.

\section{CONCLUSION}

We simulate tidal current of $\mathrm{M}_{2}$ component tide in Jiaozhou Bay at first, the results conform to the tidal theory and probably conform to the measured values of the flow measurement report of crude oil pier Phase III at Qingdao Harbor. Then, we proposes an approach to implement the 3D visualization of oil spill, oil can move with wave model and melt into one another. The $3 \mathrm{D}$ visualization is in real-time, but the speed of calculating tidal current is slow, so there are a lot of works to do in improving the efficiency of tidal current algorithm.

\section{ACKNOWLEDGEMENT}

We would like to thank the anonymous reviewers for their helpful suggestions and comments and also thank the support by the 973 Program of China (No. 2002CB312103).

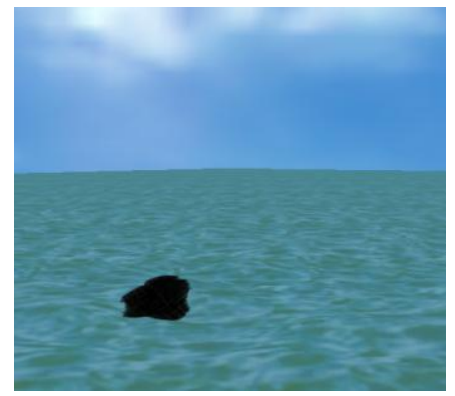

Fig. 9. Initial status.

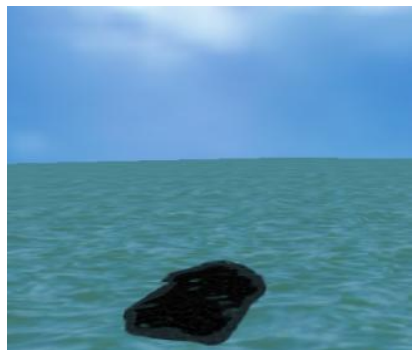

Fig. 10. After 20 minutes.

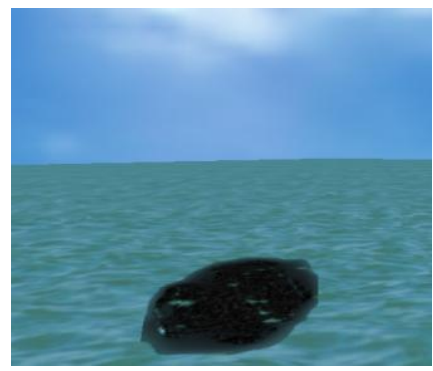

Fig. 11. After 30 minutes.

\section{REFERENCES}

[1] A. F. Blumberg and G. L. Mellor. A description of a three-dimensional coastal ocean circulation model, Three Dimensional Coastal Ocean Models, vol.4, pp.1-16, 1987.

[2] G. Fang, Z. Wei and Y. Wang. Development of Tide and Tidal Current Regional Prediction in China, Advances in Earth Science, vol.23, no.4, pp.331-336, Apr 2008.

[3] G. Fang, Z. Wei and B. CHOI. Interbasin Freshwater, Heat and Salt Transport Through the Boundaries of the East and South China Seas from a Variable-grid Global Ocean Circulation Model, Science in China(Series D), vol.46, no.2, pp.149-161, 2003.

[4] X. Zhou, W. Sun and H. Wang. Numerical Simulations of Lagrangian Circulation in the East China Sea I: A Self-Adaptive Circulation Model, Journal of Ocean University of Qingdao, vol.34, no.4, pp.523-528, 2004.

[5] D. W. Peachman and H. H. Rachford. The numerical solution of parabolic and elliptic differential equations, J. Soc.Industrial Appl.Math, vol.3, no.1, 1955 .

[6] Z. Huang, L. Huang. Tidal Theory and Calculation, China Ocean University Press, pp.168-181, 2005.

[7] Y. Liu, S. Yuan and J. Lin. Development and Research of Geographical Information System of Marine Oil Spill Emergency Response, Journal of Dalian Maritime University, vol.27, no.2, pp.42-45, 2001.

[8] C. Xie, Y. Jin, Z. Li and X.Liu. Improved Real-time Ocean Wave Mesh Model, Journal of System Simulation, vol.16, no.10, pp.357-360, 2004.

[9] J. A. FAY. The Spread of Oil Slicks on a Calm Sea, oil on the sea, Plenum, New York, 53-63, 1969

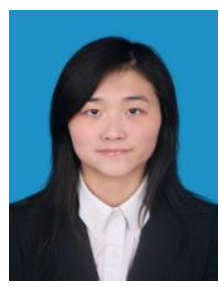

Feng Yu was born in Yunnan, China in 1982. She began $\mathrm{PhD}$ programs in 2006. Now, she is a $\mathrm{PhD}$. student in Key Laboratory of Marine Simulation and Control of Dalian Maritime University. Her main research interest is real-time marine simulation.

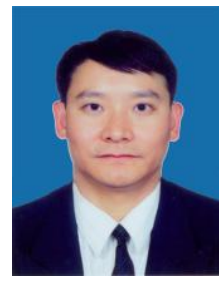

Prof. Yong Yin was born in 1969. He received his B.S. and M.S. degree in navigation technology from Dalian Maritime University in 1991 and 1994 respectively. In 2001, he received $\mathrm{PhD}$ in Transportation Information Engineering and Control from Dalian Maritime University. His research interests include simulation technology, virtual reality and computer graphi. 